\title{
Evolution of light-like Wilson loops with a self-intersection in loop space
}

\author{
T. Mertens, P. Taels \\ Departement Fysica, Universiteit Antwerpen, B-2020 Antwerpen, Belgium
}

\section{A R T I C L E I N F O}

\section{Article history:}

Received 5 September 2013

Received in revised form 18 October 2013

Accepted 6 November 2013

Available online 12 November 2013

Editor: L. Alvarez-Gaumé

\section{Keywords:}

Wilson loops

Loop space

\begin{abstract}
A B S T R A C T
Recently, we proposed a general evolution equation for single quadrilateral Wilson loops on the lightcone. In the present work, we study the energy evolution of a combination of two such loops that partially overlap or have a self-intersection. We show that, for a class of geometric variations, the evolution is consistent with our previous conjecture, and we are able to handle the intricacies associated with the self-intersections and overlaps. This way, a step forward is made towards the understanding of loop space, with the hope of studying more complicated structures appearing in phenomenological relevant objects, such as parton distributions.
\end{abstract}

(C) 2013 Elsevier B.V. All rights reserved.

\section{Introduction}

A reformulation of the Ambrose-Singer theorem in a gauge theory context [1] states that the holonomy variables $U_{\Gamma}$ of the (gauge-)connection $\mathcal{A}_{\mu}=A_{\mu}^{a} t^{a}$ :

$U_{\Gamma_{i}}=\Phi\left(\Gamma_{i}\right)=\mathcal{P} \exp \left[i g \oint_{\Gamma_{i}} d z^{\mu} \mathcal{A}_{\mu}(z)\right]$,

where the $\Gamma_{i}$ are loops, and where $t^{a}$ are the generators of the Lie algebra of the gauge group in a certain representation (here the fundamental representation of $S U\left(N_{c}\right)$ ), contain the same information as the corresponding gauge theory. From this holonomy, which is some $N \times N$ matrix in the representation of the gauge group, a gauge invariant variable can be obtained by taking the trace. ${ }^{1}$ This trace introduces, however, extra constraints on the loops: the so-called Mandelstam constraints [1-4], which assure that the product of traces over holonomies is again the trace over a holonomy. Gauge theory can then be represented in a loop space setting, where the observables are now built from the vacuum expectation values of products of traces over holonomies, referred to as Wilson loop variables [2,3,5-7]:

$\mathcal{W}_{n}\left[\Gamma_{1}, \ldots, \Gamma_{n}\right]=\left\langle 0\left|\mathcal{T} \frac{1}{N_{c}} \operatorname{Tr} \Phi\left(\Gamma_{1}\right) \cdots \frac{1}{N_{c}} \operatorname{Tr} \Phi\left(\Gamma_{n}\right)\right| 0\right\rangle$.

E-mail addresses: tom.mertens@uantwerpen.be (T. Mertens) pieter.taels@uantwerpen.be (P. Taels).

${ }^{1} U_{\Gamma}$ transforms under a gauge transformation as $U_{\Gamma}^{g}=g_{x}^{-1} U_{\Gamma} g_{x}$, where $x=$ $\Gamma(0)$. The cyclicity of the trace assures that $\operatorname{Tr} U_{\Gamma}$ is gauge invariant.
In a previous paper [8], we calculated a quadrilateral Wilson loop on the light-cone to leading order, and introduced a new differential operator:

$\frac{d}{d \ln \sigma}=s \frac{d}{d s}+t \frac{d}{d t}$

with $s$ and $t$ the Mandelstam variables (see Section 2). This operator was then used to derive an evolution equation for this class of Wilson loops, and inspired us to formulate a conjecture for a general evolution equation:

$\lim _{\epsilon \rightarrow 0} \mu \frac{d}{d \mu}\left(\frac{d}{d \ln \sigma} \ln \mathcal{W}_{1}(\Gamma)\right)=-\sum_{\text {cusps }} \Gamma_{\text {cusp }}$,

where $\epsilon$ is defined in the dimensional regularization procedure $D=4-2 \epsilon$. It was also shown that the evolution of the cusp and $\Pi$-shape configurations is consistent with this conjecture (see [8] for the details). It turns out that the operator, Eq. (4), is a special case of the Fréchet derivative [9,10], the details of which will be discussed elsewhere [11].

In this work, we consider some symmetrical combinations of two quadrilateral Wilson loops on the light-cone, for which we test conjecture (4). Put in a Wilson loop variable language: we are calculating $\mathcal{W}_{1}[\Gamma]$, with $\Gamma=\Gamma_{1} \Gamma_{2}$, where the product between the loops is defined in generalized loop space [12]. Important is that these Wilson loop configurations exhibit intricacies, associated with the self-intersection and overlap, that usually cause problems in a loop space approach. A close inspection of these intricacies indicate that one needs to be careful in counting cusps along the path. We show that the effective number of cusps can deviate from the number one would expect from a naive counting procedure. 


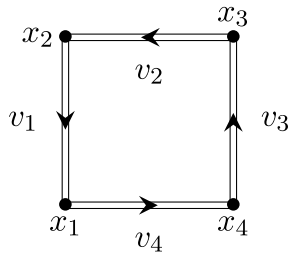

Fig. 1. Parametrization of the Wilson Loop in coordinate space.

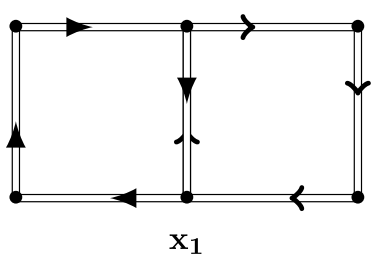

Fig. 2. Configuration 1 - overlap, equal orientation.

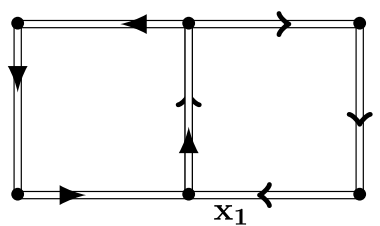

Fig. 3. Configuration 2 - overlap, opposite orientation.

Furthermore, the group structure of loop space is confirmed by our explicit calculations.

\section{Loops and parametrization}

We combine two similar quadrilateral Wilson loops on the light-cone in two different configurations: with a partial overlap (Figs. 2 to 3), and with a self-intersection (Figs. 4 to 5). Each loop is parametrized by four vectors $v_{i}$ on the light-cone (i.e. $v_{i}^{2}=0, \forall i$ ), as shown in Fig. 1. In the symmetric cases under consideration, both loops are equal in size and hence can be parametrized by these four vectors. We also introduce the Mandelstam variables ${ }^{2}$

$s=\left(v_{1}+v_{2}\right)^{2}=2 v_{1} v_{2}, \quad t=\left(v_{2}+v_{3}\right)^{2}=2 v_{2} v_{3}$,

in order to simplify the notation of the results of the calculation. Each configuration has two different possible relative orientations for the constituting loops: one where the orientation is equal (Figs. 2 and 5) and one where the orientation is opposite (Figs. 3 and 4). These orientations define how the Wilson line - gluon vertices are ordered along the path, and also fix the color flow at the self-intersection or overlap. In the figures, the color flow along the loops is represented by the different arrow styles, and the point $\mathbf{x}_{1}$ represents the base-point of the considered loop space. Here we only consider color neutral objects, in other words: there are no gluons in the initial or final state.

\section{Loop-group structure}

Expanding Eq. (1) to second order

$\Phi(\Gamma)=1+i g \oint_{\Gamma} d z^{\mu} \mathcal{A}_{\mu}(z)-\frac{g^{2}}{2 !} \mathcal{P} \oint_{\Gamma} d z^{\mu} d z^{\prime}{ }^{\nu} \mathcal{A}_{\mu}(z) \mathcal{A}_{\nu}\left(z^{\prime}\right)$,

\footnotetext{
2 Note the signs!
}

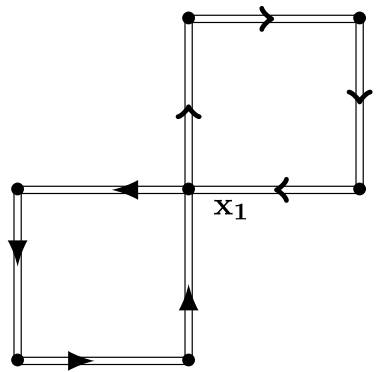

Fig. 4. Configuration 3 - self-intersection, opposite orientation.

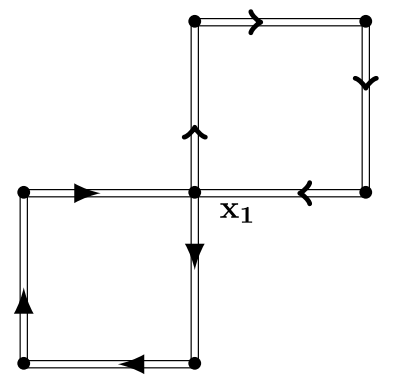

Fig. 5. Configuration 4 - self-intersection, equal orientation.

and taking traces and vacuum expectation values yields:

$\mathcal{W}_{1}(\Gamma)=1-\frac{g^{2}}{2 !} \operatorname{Tr}\left(t^{a} t^{b}\right)\left\langle 0\left|\mathcal{T} \oint_{\Gamma} d z^{\mu} d z^{\prime}{ }^{\nu} A_{\mu}^{a}(z) A_{v}^{b}\left(z^{\prime}\right)\right| 0\right\rangle$,

for the first order Wilson loop variable. The generators and gauge connections in Eq. (7) are ordered along the loop by the timeordering operation $\mathcal{T}$, where the "time" is represented by the path parameter $t \in[0,1]$ such that $d z^{\mu}=\dot{z}^{\mu} d t$. Now, considering $\Gamma=\Gamma_{1} \Gamma_{2}$, the group structure of generalized loop space [12-15] allows us to rewrite the integral in the second term of Eq. (7) as

$\oint_{\Gamma_{1} \Gamma_{2}} \mathcal{A}_{\mu} \mathcal{A}_{v}=\oint_{\Gamma_{1}} \mathcal{A}_{\mu} \mathcal{A}_{v}+\oint_{\Gamma_{1}} \mathcal{A}_{\mu} \oint_{\Gamma_{2}} \mathcal{A}_{v}+\oint_{\Gamma_{2}} \mathcal{A}_{\mu} \mathcal{A}_{\nu}$

where $\mathcal{A}_{\mu}$ and $\mathcal{A}_{v}$ are again ordered along the path ${ }^{3}$ and the integral measures are suppressed. Eq. (8) makes it clear that there are three contributions: two coming from the loops considered independently, and one coming from the interference terms. Also, the group structure of generalized loop space ${ }^{4}$ takes care of what happens when one changes the orientation of one of the two loops:

$$
\begin{aligned}
& \oint_{\Gamma_{1} \Gamma_{2}^{-1}} \mathcal{A}_{\mu} \mathcal{A}_{v} \\
& =\int_{\Gamma_{1}} \mathcal{A}_{\mu} \mathcal{A}_{v}+\int_{\Gamma_{1}} \mathcal{A}_{\mu} \int_{\Gamma_{2}^{-1}} \mathcal{A}_{v}+\int_{\Gamma_{2}^{-1}} \mathcal{A}_{\mu} \mathcal{A}_{v} \\
& =\int_{\Gamma_{1}} \mathcal{A}_{\mu} \mathcal{A}_{v}+(-1)^{1} \int_{\Gamma_{1}} \mathcal{A}_{\mu} \int_{\Gamma_{2}} \mathcal{A}_{v}+(-1)^{2} \int_{\Gamma_{2}} \mathcal{A}_{v} \mathcal{A}_{\mu} .
\end{aligned}
$$

In the last term, the order of the algebra generators inside the trace needs to be reversed as well (i.e. $\operatorname{Tr}\left(t^{a} t^{b}\right) \rightarrow \operatorname{Tr}\left(t^{b} t^{a}\right)$ ). However, due to the cyclicity of the trace, at one-loop level both traces

\footnotetext{
3 This means we do not need to consider the contribution $\int_{\Gamma_{1}} \mathcal{A}_{v} \int_{\Gamma_{2}} \mathcal{A}_{\mu}$.

4 The group structure was confirmed by explicit calculation of the full diagrams.
} 


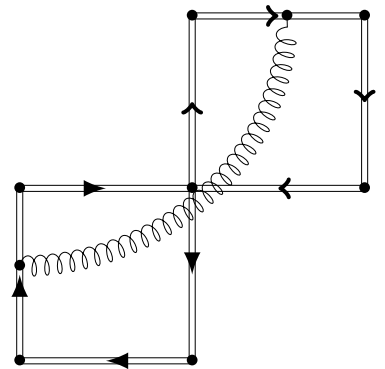

Fig. 6. Configuration 4: example of an interference diagram.

yield the same result. Moreover, in the first order, the value of the Wilson loop variables of the single loops: the first and last term in (8), are independent of the orientation of the loop. Therefore, we can immediately write down the single loop contributions to Eq. (8) and to Eq. (9) from our previous results in [8] (see Eq. (11)).

\section{Diagram results}

Using the dimensionally regularized gluon propagator in the Feynman gauge and in coordinate representation:

$D_{\mu \nu}^{a b}\left(z-z^{\prime}\right)=\frac{\left(\mu^{2} \pi\right)^{\epsilon}}{4 \pi^{2}} \Gamma[1-\epsilon] g_{\mu \nu} \delta^{a b} \frac{1}{\left(-\left|z-z^{\prime}\right|^{2}-i 0\right)^{1-\epsilon}}$,

we obtain for a single quadrilateral loop on the light-cone [8, 16-18]:

$$
\begin{aligned}
\mathcal{W}_{1}\left(\Gamma_{1}\right)= & \mathcal{W}_{1}\left(\Gamma_{2}\right) \\
\approx & 1-\frac{N_{c} \alpha_{s} \pi^{\epsilon}}{2 \pi} \Gamma[1-\epsilon] \\
& \times\left(\frac{1}{\epsilon^{2}}\left(-s \mu^{2}\right)^{\epsilon}+\frac{1}{\epsilon^{2}}\left(-t \mu^{2}\right)^{\epsilon}-\frac{1}{2} \ln \left(\frac{s}{t}\right)^{2}+\text { finite }\right) \\
& +\mathcal{O}\left(\alpha_{s}^{2}\right),
\end{aligned}
$$

where we took the large $N_{c}$ limit [3,19-21] and assumed that $\Gamma_{1}$ and $\Gamma_{2}$ are equal in size. The interference terms of Eq. (7) are computed as follows: let us consider, for example, one of the diagrams that contribute to the interference term of configuration 4 (Fig. 5), which is shown in Fig. 6. In order to calculate this diagram, we parametrize the coordinates $z$ and $z^{\prime}$ from the gluon propagator along the relevant parts of the path as follows:

$z=x_{1}-v_{1}-x v_{2}, \quad x \in[0,1]$,

$z^{\prime}=x_{1}+v_{2}+(1-y) v_{1}, \quad y \in[0,1]$,

so that the denominator of gluon propagator (10) becomes:

$\left(-\left(z-z^{\prime}\right)^{2}\right)^{1-\epsilon}=\left(-2 v_{1} v_{2}(1+x)(2-y)\right)^{1-\epsilon}$.

The integral measure $d z d z^{\prime}$ in this case becomes $v_{1} v_{2} d x d y=$ $\frac{s}{2} d x d y$, hence:

$$
\begin{aligned}
- & \frac{C_{F}}{N_{c}} \frac{g^{2}}{2} \int_{\Gamma_{1}} A_{\mu}(z) d z \int_{\Gamma_{2}} A_{\nu}\left(z^{\prime}\right) d z^{\prime} \\
= & -\frac{C_{F}}{N_{c}} \frac{g^{2}}{2} \frac{\left(\mu^{2} \pi\right)^{\epsilon}}{4 \pi^{2}} \Gamma[1-\epsilon] \\
& \times \frac{1}{2} \int_{0}^{1} \int_{0}^{1} d x d y \frac{(-s)}{(-s(1+x)(2-y))^{1-\epsilon}}
\end{aligned}
$$

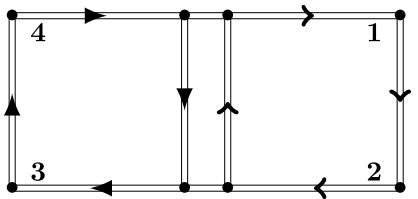

Fig. 7. Configuration 1 reduced cusps (with overlapping paths separated).

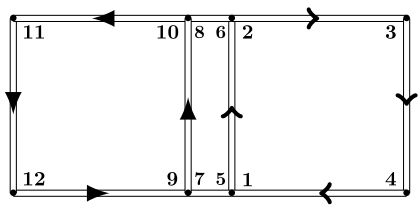

Fig. 8. Configuration 2 extra cusps (with overlapping paths separated).

$$
\approx-\frac{N_{c} \alpha_{s} \pi^{\epsilon}}{2 \pi} \Gamma[1-\epsilon] \frac{1}{2}(-s)^{\epsilon} \frac{1}{\epsilon^{2}}\left(2^{\epsilon}-1\right)^{2},
$$

in the large $N_{c}$ limit. Similar calculations can be done for the other diagrams and configurations. After summing all the contributions, this results in the following expressions for the considered configurations in the large $N_{c}$ limit (the index refers to the configuration number):

$$
\begin{aligned}
& {\left[\left\langle 0\left|\oint_{\Gamma_{1} \Gamma_{2}} A_{\mu} A_{\nu}\right| 0\right\rangle\right]_{1}} \\
& \approx \frac{N_{c} \alpha_{s}\left(\pi \mu^{2}\right)^{\epsilon}}{2 \pi} \Gamma[1-\epsilon] \frac{1}{\epsilon^{2}} \\
& \times\left((-s)^{\epsilon}+(-t)^{\epsilon}-\left(2^{\epsilon}-1\right)\left[(-t)^{\epsilon}+(-s)^{\epsilon}\right]\right) \\
& + \text { finite }+\mathcal{O}(\epsilon) \text {, } \\
& {\left[\left\langle 0\left|\oint_{\Gamma_{1} \Gamma_{2}^{-1}} A_{\mu} A_{\nu}\right| 0\right\rangle\right]_{2}} \\
& \approx-\frac{N_{c} \alpha_{s}\left(\pi \mu^{2}\right)^{\epsilon}}{2 \pi} \Gamma[1-\epsilon] \frac{1}{\epsilon^{2}} \\
& \times\left((-s)^{\epsilon}+(-t)^{\epsilon}-\left(2^{\epsilon}-1\right)\left((-t)^{\epsilon}+(-s)^{\epsilon}\right)\right) \\
& + \text { finite }+\mathcal{O}(\epsilon) \text {, } \\
& {\left[\left\langle 0\left|\oint_{\Gamma_{1} \Gamma_{2}^{-1}} A_{\mu} A_{\nu}\right| 0\right\rangle\right]_{3}} \\
& \approx \frac{N_{c} \alpha_{s}\left(\pi \mu^{2}\right)^{\epsilon}}{2 \pi} \Gamma[1-\epsilon] \frac{1}{\epsilon^{2}} \\
& \times\left((-s)^{\epsilon}-2(-s)^{\epsilon}\left(2^{\epsilon}-1\right)+(-s)^{\epsilon}\left(2^{\epsilon}-1\right)^{2}\right) \\
& + \text { finite }+\mathcal{O}(\epsilon) \\
& {\left[\left\langle 0\left|\oint_{\Gamma_{1} \Gamma_{2}} A_{\mu} A_{\nu}\right| 0\right\rangle\right]_{4}} \\
& \approx-\frac{N_{c} \alpha_{s}\left(\pi \mu^{2}\right)^{\epsilon}}{2 \pi} \Gamma[1-\epsilon] \frac{1}{\epsilon^{2}} \\
& \times\left((-s)^{\epsilon}-2(-s)^{\epsilon}\left(2^{\epsilon}-1\right)+(-s)^{\epsilon}\left(2^{\epsilon}-1\right)^{2}\right) \\
& + \text { finite }+\mathcal{O}(\epsilon) \text {. }
\end{aligned}
$$

It should be clear from Eq. (9) that, at one-loop order, the sign of the interference term changes when reversing the orientation of one of the loops, which is indeed confirmed by Eqs. (15) to (18). 


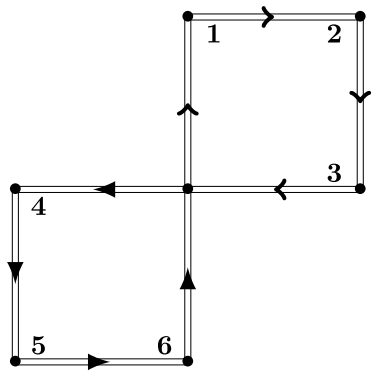

Fig. 9. Configuration 3 reduced number of cusps.

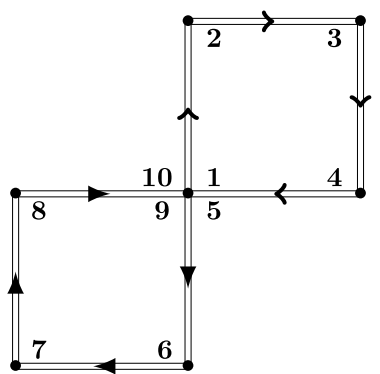

Fig. 10. Configuration 4 extra cusps.

\section{Differential operator}

Finally, we want to apply the differential operator (3) followed by the operator $\mu \frac{d}{d \mu}$, to the logarithm of the considered loop configurations, after which we take the $\epsilon \rightarrow 0$ limit. Only symmetric variations $^{5,6}$ are taken into account, in such a way that the complete loop does not change its general structure. Indeed, if one does consider structure changing variations, the number of cusps can change and in [11] we will show explicitly that there is no continuous deformation in loop space between two structurally different loops (i.e. loops with a different number of cusps). The variations we apply here are shown in Figs. 11 to 14. Applying the said combination of operators to the different configurations in the $\epsilon \rightarrow 0$ and large $N_{c}$ limits yields the following results:

$\lim _{\epsilon \rightarrow 0} \mu \frac{d}{d \mu} \frac{d \ln \left(\mathcal{W}_{1}(\Gamma)\right)_{1}}{d \ln \sigma}=-4 \Gamma_{\text {cusp }}$,

$\lim _{\epsilon \rightarrow 0} \mu \frac{d}{d \mu} \frac{d \ln \left(\mathcal{W}_{1}(\Gamma)\right)_{2}}{d \ln \sigma}=-12 \Gamma_{\text {cusp }}$,

$\lim _{\epsilon \rightarrow 0} \mu \frac{d}{d \mu} \frac{d \ln \left(\mathcal{W}_{1}(\Gamma)\right)_{3}}{d \ln \sigma}=-6 \Gamma_{\text {cusp }}$,

$\lim _{\epsilon \rightarrow 0} \mu \frac{d}{d \mu} \frac{d \ln \left(\mathcal{W}_{1}(\Gamma)\right)_{4}}{d \ln \sigma}=-10 \Gamma_{\text {cusp }}$,

where

$\Gamma_{\text {cusp }} \approx \frac{\alpha_{s} N_{c}}{2 \pi}+\mathcal{O}\left(\alpha_{s}^{2}\right)$.

Naively counting the number of cusps in the different configurations yields a total of eight, in each configuration. If this is true, however, the results contradict our conjecture Eq. (4) [8]: indeed,

\footnotetext{
5 Due to the fact that $s \frac{d}{d s}=(2 s) \frac{d}{d(2 s)}$ and $t \frac{d}{d t}=(2 t) \frac{d}{d(2 t)}$ we only need to consider $s \frac{d}{d s}+t \frac{d}{d t}$.

6 Non-symmetric variations are now being studied by us, the results will be reported separately [11].
}

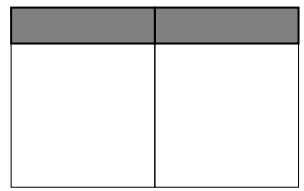

Fig. 11. $\delta t$ variation of the first two configurations.

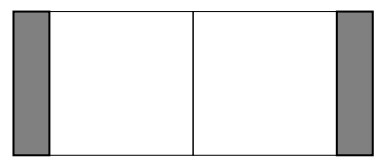

Fig. 12. $\delta s$ variation of the first two configurations

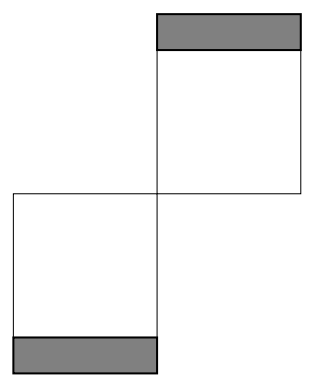

Fig. 13. $\delta t$ variation of the second two configurations.

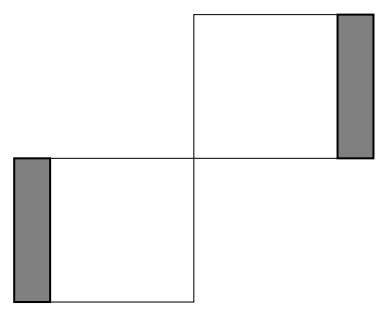

Fig. 14. $\delta s$ variation of the second two configurations.

we would expect for all the configurations a value of $-8 \Gamma_{\text {cusp }}$ in the r.h.s. of (19). To understand this apparent contradiction, one has to take a closer look at how to count the number of cusps effectively present in the studied loop. Due to path reduction [12-15], configuration 1 (Fig. 2$)^{7}$ can be reduced to a single quadrilateral so that there are effectively only four cusps (see also Fig. 7). Fig. 8 shows how count the number of cusps in configuration 2 (Fig. 3). Here the extra cusps stem from the fact that the middle line is crossed twice in the same direction by the color flow along the path, so that these four $\operatorname{cusps}^{8}$ contribute twice to the total number of cusps. Similar reasoning can be applied to configurations 3 (Fig. 4) and 4 (Fig. 5), where the counting is demonstrated in Figs. 9 and 10, respectively.

\section{Conclusions and remarks}

By explicit calculation, we have showed that the symmetric double Wilson loops obey the generalized loop space group struc-

\footnotetext{
7 We separated the overlapping paths in the figure to clarify the counting procedure.

${ }^{8}$ Four because the cusps left and right of the middle path show up in the calculations of the interference contribution.
} 
ture with respect to the inverse and the product of two loops. We also showed that the presented results are consistent with our evolution conjecture in [8], at least at leading order. At the same time, we successfully dealt with some of the intricacies, caused by a selfintersection and a partial overlap, that appear in calculations of Wilson loops as functionals in loop space. This opens the door to the calculation of more complicated structures, such as the ones appearing in the calculations of transverse dependent momentum distributions (TMDs) or soft factors in certain factorization schemes in QCD [22,23].

At first sight one would expect our conjecture (4) to only hold up to leading order, since higher order calculations will add extra terms who's behavior under this derivative is not immediately clear. In fact the derivative we used is only the lowest order part of a general differential operator known as the Frećhet derivative. The expansion of this operator and explicit demonstration of its reduction to the differential operator we used to derive our conjecture will be reported separately.

We would also like to point out that, when considering planar Wilson loops on the light-cone, the number of cusps seem to behave like a winding number of some sort, in the sense that in some cases the cusps add, and in other cases they subtract. Also note that from a loop space point of view, loops on the light-cone with a different number of cusps cannot be deformed continuously into each other [11], inducing a kind of connected component structure in loop space. These considerations suggests a possible connection with the logarithm in the complex plane, where the number of times that one winds around the origin could be interpreted as a representation of the solution branch on which one is located. In our case, this winding would be represented by the number of cusps, while the different connected components represent the different solution branches. This "duality" will be reported separately.

\section{Acknowledgements}

We would like to thank Igor Cherednikov and Frederik Van der Veken for useful discussions and suggestions.

\section{References}

[1] R. Giles, Phys. Rev. D 24 (1981) 2160.

[2] R.A. Brandt, A. Gocksch, M. Sato, F. Neri, Phys. Rev. D 26 (1982) 3611

[3] Y. Makeenko, A.A. Migdal, Phys. Lett. B 88 (1979) 135.

[4] S. Mandelstam, Phys. Rev. 175 (1968) 1580.

[5] L.F. Alday, B. Eden, G.P. Korchemsky, J. Maldacena, E. Sokatchev, J. High Energy Phys. 1109 (2011) 123, arXiv:1007.3243 [hep-th].

[6] L.F. Alday, J.M. Maldacena, J. High Energy Phys. 0706 (2007) 064, arXiv:0705.0303 [hep-th].

[7] Y. Makeenko, A.A. Migdal, Nucl. Phys. B 188 (1981) 269.

[8] I.O. Cherednikov, T. Mertens, F.F. Van der Veken, Phys. Rev. D 86 (2012) 085035 , arXiv:1208.1631 [hep-th].

[9] J. Munkres, Analysis on Manifolds, Advanced Book Classics, Addison-Wesley Publishing Company, Advanced Book Program, 1991.

[10] J. Dieudonne, Foundations of Modern Analysis, Pure and Applied Mathematics, Read Books, 2008.

[11] I.O. Cherednikov, T. Mertens, P. Taels, 2013, in preparation.

[12] J.N. Tavares, Int. J. Mod. Phys. A 9 (1994) 4511, arXiv:hep-th/9305173.

[13] K.T. Chen, Trans. Am. Math. Soc. 89 (1958) 395.

[14] K.T. Chen, J. Algebra 9 (1968) 8.

[15] K.T. Chen, Trans. Am. Math. Soc. 156 (1971) 359.

[16] I.A. Korchemskaya, G.P. Korchemsky, Phys. Lett. B 287 (1992) 169.

[17] A. Bassetto, I. Korchemskaya, G. Korchemsky, G. Nardelli, Nucl. Phys. B 408 (1993) 62, arXiv:hep-ph/9303314

[18] G.P. Korchemsky, J.M. Drummond, E. Sokatchev, Nucl. Phys. B 795 (2008) 385, arXiv:0707.0243 [hep-th].

[19] Y. Makeenko, Phys. At. Nucl. 73 (2010) 878, arXiv:0906.4487 [hep-th].

[20] Y. Makeenko, Acta Phys. Pol. B 39 (2008) 3047, arXiv:0810.2183 [hep-th].

[21] Y. Makeenko, arXiv:hep-th/0407028, 2004.

[22] C.J. Bomhof, P.J. Mulders, Nucl. Phys. B 795 (2008) 409, arXiv:0709.1390 [hep-ph].

[23] I. Cherednikov, T. Mertens, P. Taels, F.F. Van der Veken, arXiv:1308.3116 [hep-ph], 2013. 